\title{
Groping for quantitative digital 3-D image analysis: an approach to quantitative fluorescence in situ hybridization in thick tissue sections of prostate carcinoma
}

\author{
Karsten Rodenacker, Michaela Aubele, Peter Hutzler and P.S. Umesh Adiga* \\ GSF National Research Center for Environment and Health, Institute of Pathology, \\ Ingolstädter Landstr. 1, D-85764 Neuherberg, Germany \\ Tel: +49(0) 893187 3401; Fax: +49(0) 893187 3349; Email: rodena@gsf.de
}

Received 10 February 1997

Revised 28 May 1997

Accepted 20 June 1997

\begin{abstract}
In molecular pathology numerical chromosome aberrations have been found to be decisive for the prognosis of malignancy in tumours. The existence of such aberrations can be detected by interphase fluorescence in situ hybridization (FISH). The gain or loss of certain base sequences in the desoxyribonucleic acid (DNA) can be estimated by counting the number of FISH signals per cell nucleus. The quantitative evaluation of such events is a necessary condition for a prospective use in diagnostic pathology. To avoid occlusions of signals, the cell nucleus has to be analyzed in three dimensions. Confocal laser scanning microscopy is the means to obtain series of optical thin sections from fluorescence stained or marked material to fulfill the conditions mentioned above.

A graphical user interface (GUI) to a software package for display, inspection, count and (semi-)automatic analysis of 3-D images for pathologists is outlined including the underlying methods of 3-D image interaction and segmentation developed. The preparative methods are briefly described. Main emphasis is given to the methodical questions of computer-aided analysis of large 3-D image data sets for pathologists. Several automated analysis steps can be performed for segmentation and succeeding quantification.

However tumour material is in contrast to isolated or cultured cells even for visual inspection, a difficult material. For the present a fully automated digital image analysis of 3-D data is not in sight. A semi-automatic segmentation method is thus presented here.
\end{abstract}

Keywords: Fluorescence in situ hybridization (FISH), confocal laser scanning microscopy, graphical user interface, 3-D image analysis, segmentation

\footnotetext{
${ }^{*}$ Guest scientist, from CVPR Unit, Indian Statistical Institute, Calcutta, India.
} 


\section{Introduction}

\subsection{Molecular pathology}

Prostate cancer is currently a leading cause of deaths from malignancies in American and European men [11]. Owing to improved methods of detection, the number of cases being reported has increased sharply. The clinical course of prostate cancer is often unpredictable, and various efforts have been undertaken to improve reliability of prognostic parameters. Morphological grading, staging, and tumour size are mainly used to assess the malignant potential of prostate cancer [7]. Besides these methods, new techniques like nucleic acid fluorescence in situ hybridization (FISH) have become increasingly important in diagnostic and research pathology. Using FISH numerical chromosome aberrations, e.g., gain or loss of specific single chromosomes (e.g., trisomy or monosomy) can be detected. Several studies have shown $[2,8]$ that trisomy of chromosome 7 is found in malignant and non-malignant tumours of lung, kidney, and brain, as well as in tumours of the prostate. Considering that the biological significance of this alteration is still under discussion Zitzelsberger et al. [18] have shown that trisomy of chromosome 7 may be used as a prognostic factor because of the correlation with the development of lymph node metastases and grade of differentiation. Therefore, an improved image analysis procedure for quantitative evaluation of FISH in sections of prostate cancer has been established to simplify its quantitative evaluation, to confirm its prognostic value as well as to correlate FISH data with clinical follow-up.

\subsection{The need for 3-D FISH signal count}

A reliable count of FISH signals in tumour tissue necessitates a clear recognition of the tumour cells. This is only possible in sections with recognisable morphology. Standard histological sections contain only few cell nuclei completely inside the section. Only for those nuclei, a reliable count of FISH signals can be assured [1], hence thick sections have to be analysed for sufficient number of cells.

However, such thick sections cannot be analysed in 2-D because of the degraded image quality and of the possible and frequent occlusion of FISH signals. The latter can be suspected from the relatively stable amount of monosomies mostly independent of the number of tri- and polysomies [16].

In addition, the analysis of 3-D images necessitates new training for the pathologists. Since the actual state of 3-D data representation (cyberspace, virtual reality) is far from routine applicability the interactive display and processing this approach has to be as near as possible to the typical observation behaviour of pathologists.

\subsection{Image acquisition, processing and analysis}

The seemingly simple task of signal counts becomes difficult under the condition that the count has to be done with respect to a single cell nucleus. When these nuclei are isolated it is easy to estimate the membership of a signal to a nucleus. However, in most tumour tissues this relation is difficult or may be impossible to determine. This is true for visual inspection and even more so for computer based analysis. It is a well known fact that segmentation is the most cumbersome task in any digital image analysis approach. Recently another graphical user interface for visual inspection was presented [3] using primarily volume techniques for display. In our approach we tried to mimic the inspection behaviour of pathologists by mainly presenting sections, whatever volume rendering is 
possible. The interactive inspection part is already used for a comparison of thin and thick section FISH count already reported in [1].

Concerning segmentation of nuclei in 3-D in $[4,15,17]$ proposed segmentation methods are mainly based on 2-D segmentation schemes, and more or less elaborated merging operations. As mentioned above, the segmentation of isolated nuclei is a minor problem. Therefore we address preferentially the problem of connected nuclei by interactive means.

\section{Material: preparation, staining and sampling}

Tissue sections: $15 \mu \mathrm{m}$ thick paraffin sections from routinely processed prostate cancer tissue were deparaffinized and rehydrated.

DNA probes and probe labeling: $\alpha$-satellite DNA probes specific for the centromeric region of chromosome 7 were generated from clone 680TA [18]. The DNA probe was labeled by nick-translation with biotin-dUTP (Boehringer, Mannheim) according standard procedure [18].

Hybridization: The fluorescence in situ hybridization (FISH) was performed according to [1]. Briefly, the slides were treated with pronase E $\left(0.05 \%\right.$ in $2 x S S C$, Merck, Darmstadt, Germany) at $37^{\circ} \mathrm{C}$ for 20 to $35 \mathrm{~min}$. After denaturation $\left(70 \%\right.$ formamide/2xSSC) at $72{ }^{\circ} \mathrm{C}$ for 20 to $45 \mathrm{~min}$, the slides were dehydrated in an ethanol series. The hybridization mix consisted of carrier DNA (herring sperm DNA, SIGMA), 55\% formamide/1xSSC, $10 \%$ dextran sulphate and the labeled DNA probe. This mixture was applied to the slides under a glass cover slip and sealed with rubber cement. After overnight incubation at $37^{\circ} \mathrm{C}$ post-hybridization washes were performed at $43{ }^{\circ} \mathrm{C}$ in three changes of $50 \%$ formamide/2xSSC, PN buffer [9], and 3\% bovine serum albumin (BSA, SIGMA).

Detection and counter stain: The biotin-labelled centromere 7 probe was detected by streptavidinfluorescein isothiocyanate (FITC) and biotinylated anti-streptavidin conjugates (Camon, Wiesbaden). The nuclei were counter stained with propidium iodide (PI, SIGMA) and mounted with an antifade solution as described by [5]. Staining procedure was performed according to [14].

Sampling: Representative tumour and non-tumour control regions with unambiguous FISH signals and well-preserved nuclei morphology were selected for acquisition by CLSM.

\section{Methods}

The development and application of image analysis procedures are performed under the software package IDL (Interactive Data Language, Research Systems Inc., Boulder, CO, USA). As hardware development platform, a Silicon Graphics work station INDY, is used with 128 MB main memory, true color display, connected via local area network to other computers and PC's, especially the PC attached to the laser scanning microscope (LSM 410, Zeiss, Oberkochen, BRD).

Beside mere memory problems with 3-D images of sizes about $15 \mathrm{MB}$ pixels, even the processing time increases considerably. For example, even a RISC processor of MIPS 4400 type is slow for methods usually applied and well known in 2-D image analysis. 


\subsection{Image acquisition}

Fluorescence images are scanned using a Confocal Laser Scanning Microscope (CLSM) Zeiss LSM 410. Essential setup features for the acquisition of FISH signals in tissue sections are as follows: Lens Zeiss PNF $100 \times, 1.3$, zoom $=2$, realized by scanning unit). The scanned field of $62.5 \times 62.5 \mu \mathrm{m}$ is sampled to $256 \times 256$ pixels giving a pixel size of $0.25 \mu \mathrm{m}$ in $x$ and $y$ direction. Excitation laser lines are selected according to the fluorochromes used. For propidium iodide (PI) used as a DNA counter stain and FITC labeled signals both are excited by the argon line $488 \mathrm{~nm}$. The emission of both is measured simultaneously in two separate channels using a bandpass $515-565 \mathrm{~nm}$ for FITC and a longpass LP $590 \mathrm{~nm}$ for the PI channel, respectively. The axial distance selected between two subsequent confocal images depends on the further evaluation strategy. If a spatial isotropic representation of the 3-D data is intended, the axial distance is identical to the lateral distance of the pixels, i.e., $0.25 \mu \mathrm{m}$. For a $16 \mu \mathrm{m}$ thick section this results in a sequence of 64 scanned images. If only the sampling theorem should be met, roughly a $0.5 \mu \mathrm{m}$ distance is enough, keeping in mind that the confocal axial resolution of a lens with $\mathrm{NA}=1.3$ is about the wavelength, i.e., $0.6 \mu \mathrm{m}$ [13]. The FITC emission is associated with the green channel and the PI emission with the red channel of a RGB true color image. The image data are stored on disk in TIFF format. Scanning one field of view of a section with $16 \mu \mathrm{m}$ thickness and using $0.25 \mu \mathrm{m}$ distance between individual images results in an uncompressed data set of 12.58 Mbyte.

\subsection{Image display}

Various methods of display of digital volumes are proposed in this quickly growing field of methodical developments $[3,6]$. However, there are still stringent requirements in research to solve the technical as well psychological problems of display of 3-D information.

To allow an observation familiar to pathologists, a GUI has been designed (Fig. 1) displaying the 3-D image as a heap of orthogonal sections with arbitrary choice of the cutting planes in $x, y$ and $z$. Beside the counter stain volume in red colour channel and the signal in green, a second type of signal may be stored in blue colour channel. Possible display by interactions with the GUI are seen in Figs 1 and 2:

1. Orthogonal sections of the original digitized volume with counter stain in red $(\mathrm{R})$ and signal in green $(\mathrm{G})$.

2. Thresholded and segmented counter stain to improve recognition of nuclear borders with adjustable parameters.

3. Segmented signals with adjustable parameters.

4. Histograms from all channels, counter stain (HIST_R), signal (HIST_G) and blue channel (HIST_B) (Fig. 1).

5. Surface rendered counter stain images, (SLIC_R), signal (SLIC_G) and results of signal segmentation (SLIC_B) (Fig. 3).

6. Gallery of interactively selected box, sphere or ellipses around or semi-automatic segmented nuclei as a sequence of sections of sub-windows (Figs 4 and 5).

7. Journaling of the sequence of selected boxes, respectively, labelled objects including the signal count and other features. A replay function for repeated analysis of analysed locations is carried out. 
Other possible interactions for analysis are histogram based local thresholding, cell labelling, FISH signal count, global and local segmentation, cutting of globally segmented touching cell nuclei by interactively adjustable planes (knife), spheres or ellipses, where the latter can be dragged around the object of interest and then used as limit for the volume growing process, as well as active volumes for segmentation, 3-D morphological operations, etc.

\subsection{Interactive volume processing}

For interactive volume analysis the orthogonal display of the volume can be positioned in $x, y$ and $z$ direction and a box, sphere or ellipses around the object of interest resized by dragging can be selected (Fig. 4). By another mouse click the selected sub-volume is displayed in a separate window as a gallery of sub-sections (Fig. 5). On the left-hand side all sub-sections and on the right-hand side zoomed sub-sections are shown.

During display the number of FISH signals are automatically displayed inside the selected subvolume and calculated, and the mouse cursor jumps to the counting button's respective number (Fig. 2b). Clicking on the appropriate button stores the subvolume location, size and count. In the display field of orthogonal sections the box location is outlined for information of the already scanned places. The buttons SAVE, LOAD, CLEAR, REDRAW, DRAW Count and PRINT Count allow storage and display of the count list (Fig. 2b). Also old count list files can be read and redisplayed for further inspection (Fig. 6).

\subsection{Semi-automatical volume processing}

\subsubsection{Segmentation of nuclei}

The completely automatic segmentation of cell nuclei is still a distant dream. We have developed a seeded volume growing technique based on several size and shape constraints to segment the cell nuclei and to automatically count the FISH signal per nuclei. After a slight volume opening for noise reduction the image is subjected to global segmentation. It is automatically thresholded on the basis of local histograms and the 3-D connected components of the resulting two-level image are labelled. Cells which are out of focus and too complicated to segment are rejected from further evaluation. Also the cells which are at the border of the image are rejected since the completeness of such cell nuclei cannot be ascertained. Cells which are touching each other are first selected for semi-automatic segmentation. The center of such a cell is selected by clicking the mouse over the approximate centroid. This point will act as a seed and the seed is grown in all directions using the threshold derived from local histograms.

The overshoot of the marked region into neighbouring cells which touch each other is checked by the global shape constraints like maximum extension and in a second step by an adjustable ellipsoid surface which is fitted in such a way that the cell nucleus falls just within.

After separation of the touching cell nucleus, the image volume is subjected to a relabelling process to enable the newly segmented cells to have a distinct label (Fig. 7). Segmented cell regions are selected in the signal channel for further processing.

\subsubsection{Segmentation of FISH signals}

Several different methods, from thresholding via linear transformations to non-linear ones, e.g., transformations derived from mathematical morphology $[10,12]$, were applied. For the actual material 
with a small extension in $x, y$ and $z$ direction FISH signals in 3-D are represented by small grain-like structures.

A top-hat transformation was considered to be the best method to enhance the FISH signals for segmentation. The top-hat transformation image is the difference between an original grey scale image and an opened version. The radius of opening denotes the diameter of the top-hat. A top-hat threshold can be considered as the height of the top-hat. Incidently, the term top-hat is derived from 2-D image analysis where the operation can be considered as putting a top-hat on the intensity profile. This image cannot easily be transferred to 3-D situations, where the signal intensity has to be considered as a fourth dimension. The applied top-hat transformation corresponds to the simplified algorithm proposed by [16] for 2-D compact spots, where additionally, a watershed transformation is necessary to detect small neighboring spots. The latter is not necessary by applying the full 3-D top-hat transformation. All operations are performed as 3-D operations.

After thresholding the top-hat transformation, the resulting (binary) volume is processed by the selection of one connected object after the other, using again 3-D volume growing. A grain is accepted as FISH signal after a size and shape criterion and its presence located inside the labelled nucleus.

\subsection{Featuring}

The goal of quantitative FISH to analyse about pathological alterations is the count of gains and losses of chromosomes. The physiologically detectable disomic nuclei (2 signals) in tumour tissue are not of primary interest. Of most importance is the number of appearing trisomies (3 signals), polysomies ( $>3$ signals) and monosomies (1 signal). The subjective computer-aided evaluation should equip us with data on the spatial distribution of signals in the nuclei as well as about the size and shape of nuclei in 3-D.

\subsubsection{Interactive}

During visual inspection of the volume the coordinates and size of each selected box with the subjectively estimated signals are stored accompanied by the FISH signal count. This count list is subject to further analysis either by pooling count lists of several volumes or by using the box locations as expert selected seed for semi-automatical analysis.

\subsubsection{Semi-automatic}

First a global (threshold) based segmentation and object labelling is performed. The pathologist can display the result and decide which nuclei have to be counted and which are to be deleted. Selection or deletion are made by clicking on the labelled object. Improvement of segmentation is performed by selecting the object and proceeding as explained earlier under the section segmentation of nuclei. For labelled objects the FISH signals can be automatically counted, the box coordinates of the surrounding box, the count, signal and nucleus volume and the label number are stored. The FISH signals of either all labelled nuclei or only of interactively selected ones are counted. In the latter case a gallery of a box determined by the size of the selected object can be displayed, comparable to interactive processing.

Beside FISH signal count, the nuclei as well as each signal are accessible for further feature extraction such as calculation of volumes, mean total intensities and variations [4] and signal distribution in space. 


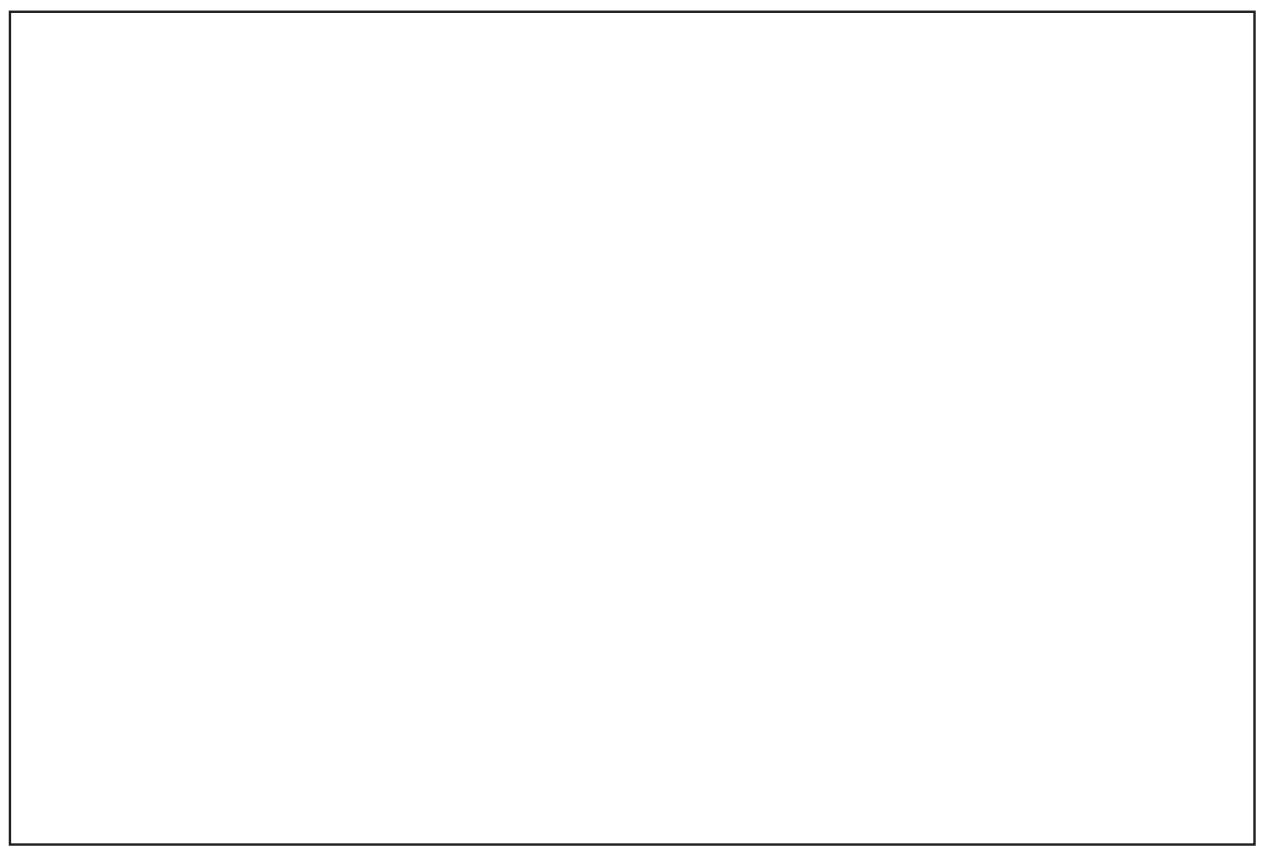

Fig. 1. Graphical user interface overview for 3-D image display.

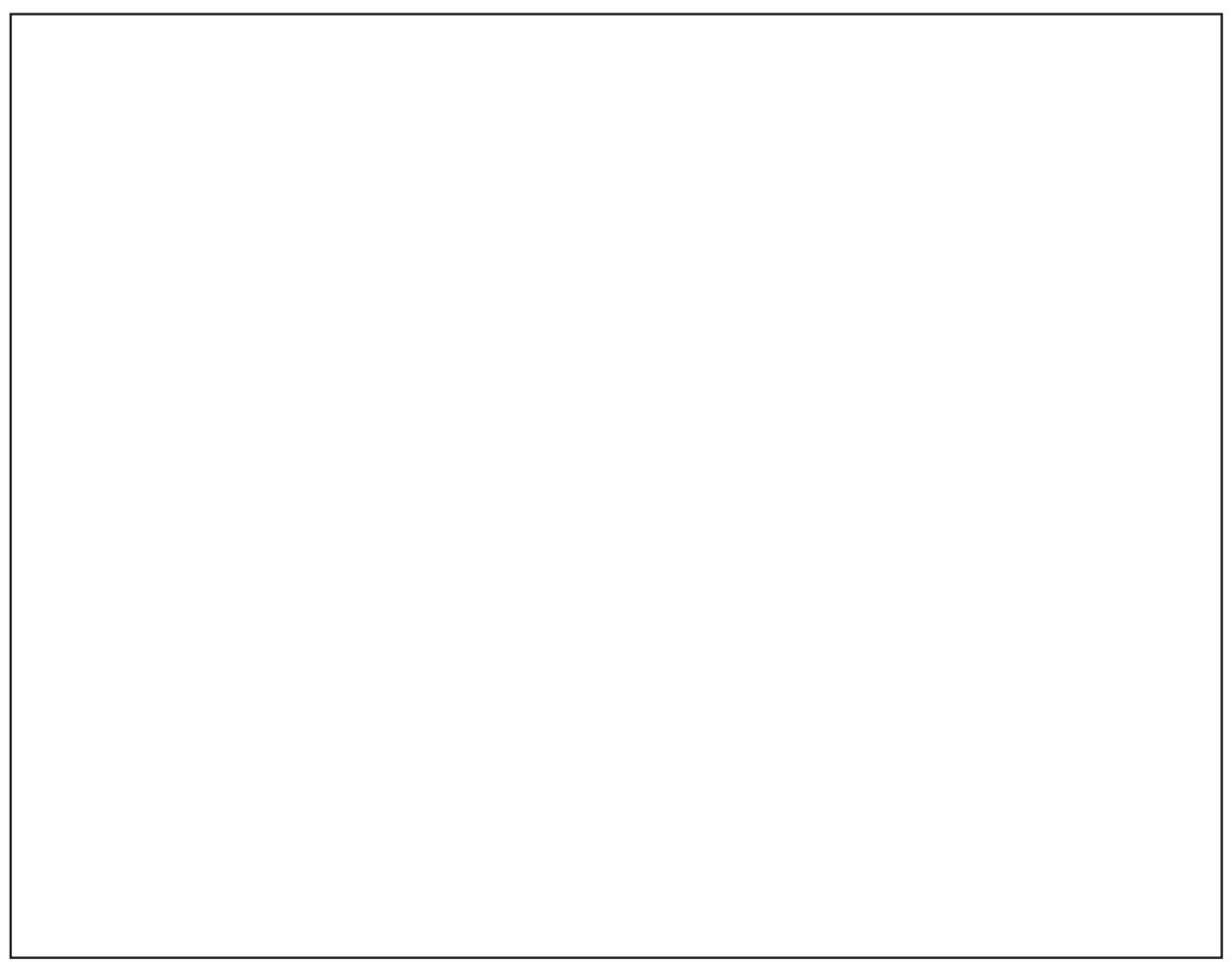

Fig. 2. Functional tablet for user interaction. (a) Segmentation and semi-automatic count and journaling. (b) Display, visual count and journaling. 
Fig. 3. Graphical user interface for volume rendering and slicing.

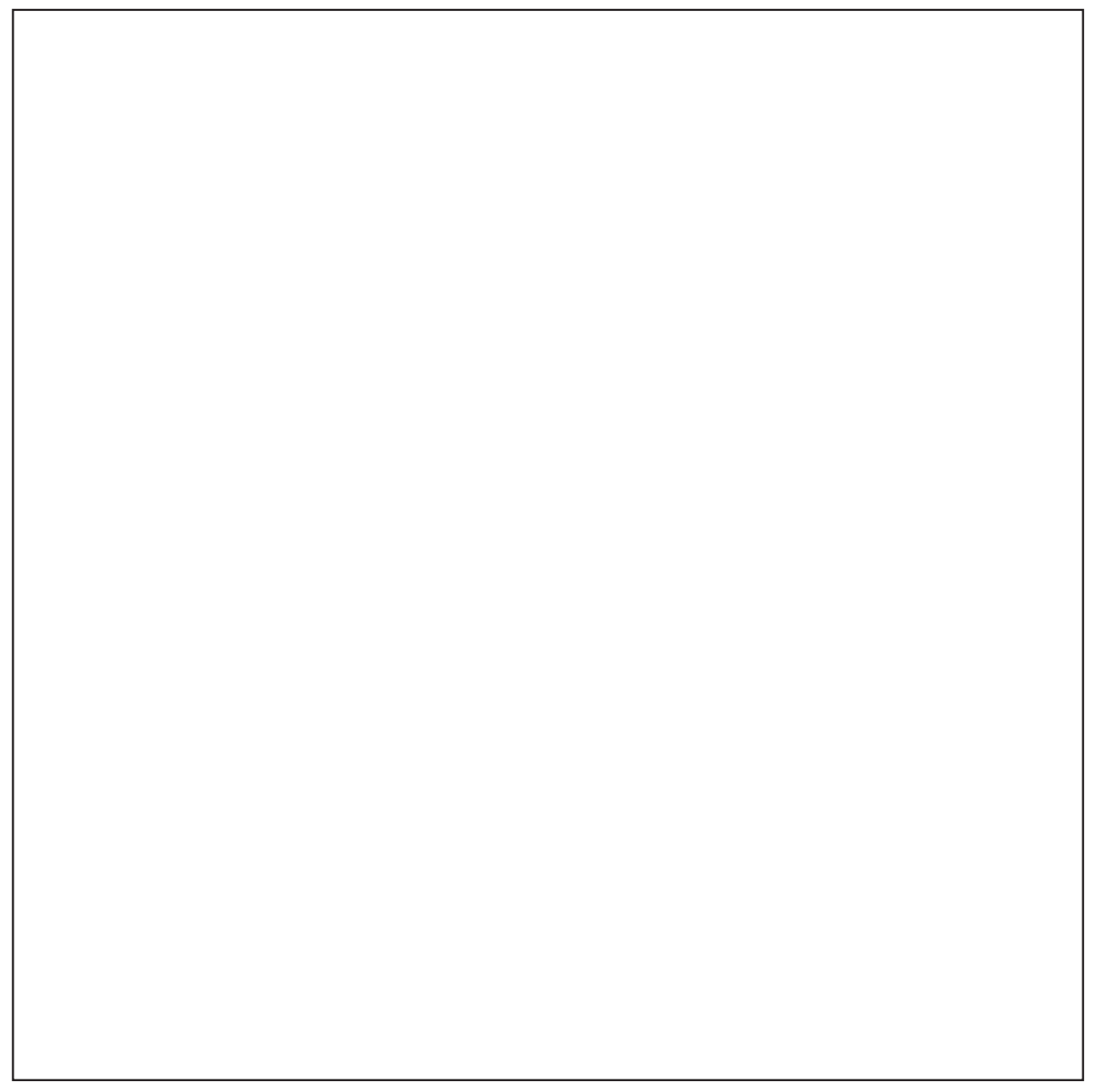

Fig. 4. Orthogonal sections in $x-y-z$ with outlined selection box. 
Fig. 5. Gallery of a selected box.

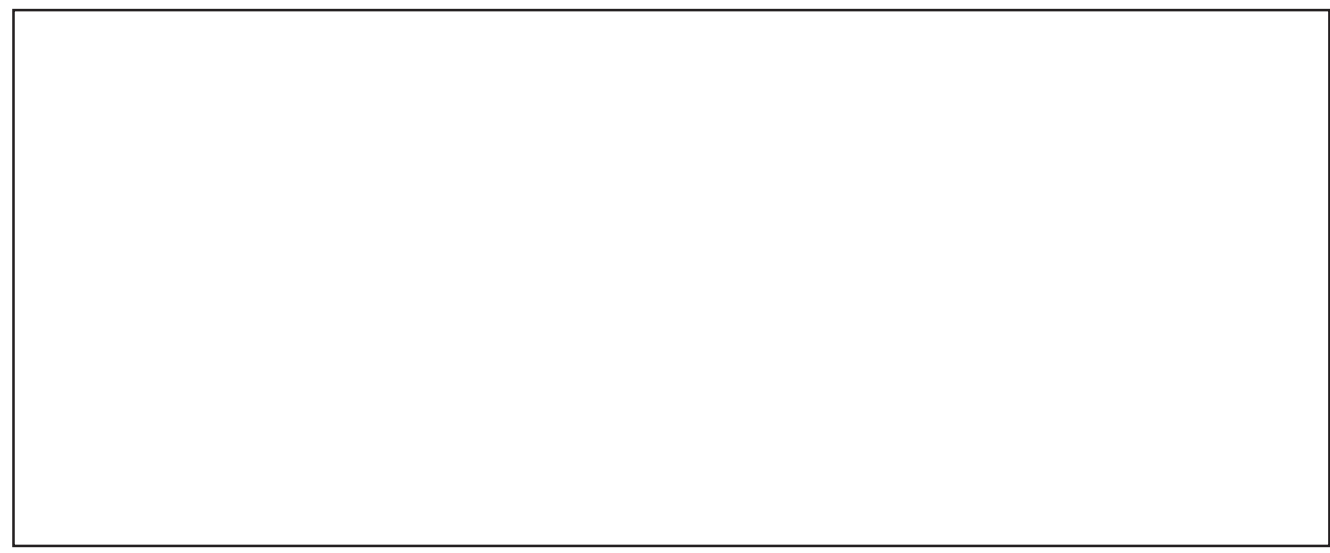

Fig. 6. Result display with (a) marked objects and (b) FISH signal distribution.

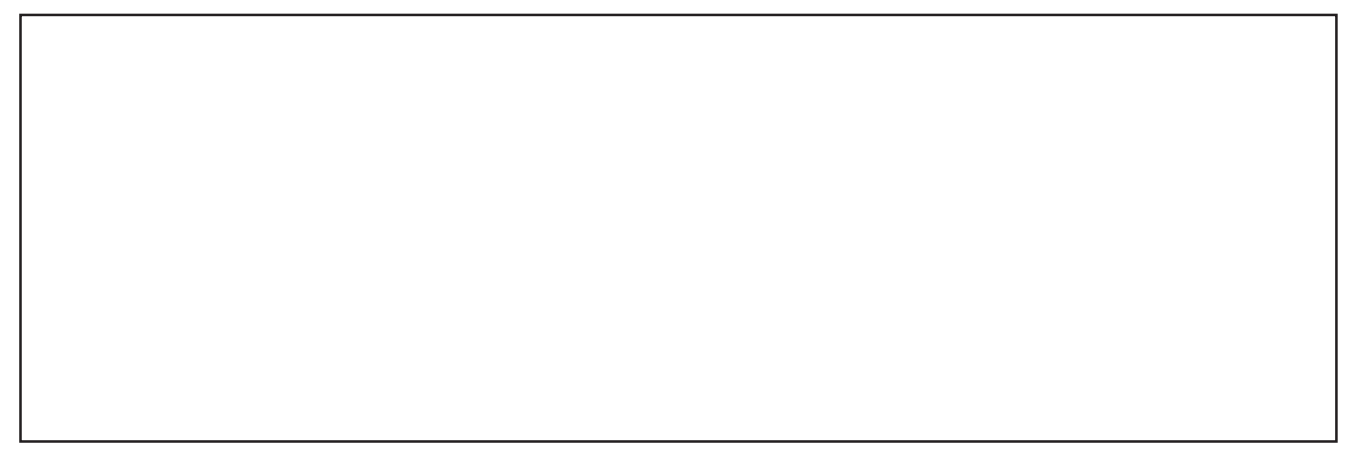

Fig. 7. Labelled section; (a) globally segmented, (b) locally improved by cutting touching nuclei, (c) cleaned by deletion. 


\section{Preliminary results and conclusions}

The interactive evaluation aid described above as well as the semi-automated evaluation, supply us with data for a more straightforward method for detecting suspected signals or ones that need to be analyzed. This would allow to concentrate the analysis steps on small regions of interest in the volume data.

Until now 19 tumours with about 300 image (volume) blocks have been visually evaluated using this program. The results presented in [1] justify the increased workload by 3-D image analysis. FISH analysis in semi-thin sections deliver a serious underestimation compared to the signal number in thick sections. The comparison of visually counted signals and semi-automatic counting in 3-D will be communicated in the near future.

The methodical proceedings for quantitative fluorescent in situ hybridization for 3-D evaluation has been outlined here and a program which can run on multiple platforms has been developed. The software is user friendly. The pathologists who are not familiar with computer aided image analysis, can easily work with this program. The software is not just for FISH evaluation but can be used for morphological feature evaluation and to work on any kind of visualizable signal in the cell nucleus or any other object. The GUI can also be used to visualize the cell distribution and/or invasive pattern of malignant cells in three dimensions.

\section{References}

[1] M. Aubele, H. Zitzelsberger, S. Szücs, M. Werner, H. Braselmann, P. Hutzler, K. Rodenacker, L. Lehmann, G. Minkus and H. Höfler, Comparative FISH analysis of numerical chromosome 7 abnormalities in $5 \mu \mathrm{m}$ and $15 \mu \mathrm{m}$ paraffinembedded tissue sections from prostatic carcinoma, Histochem. Cell Biol. 107 (1997), 121-126.

[2] V.R. Babu, B.J. Miles, J.C. Cerny, L. Weiss and D.L. van Dyke, Cytogenetic study of four cancers of prostate, Cancer Genet. Cytogenet. 48 (1990), 83-87.

[3] C.R.L. Becker, Jr., U.V. Mikel, L.T.C.W.R. Oliver and I.A. Sesterhenn, Enumeration of interphase chromosomes: Comparison of visual in situ hybridization and confocal fluorescence in situ hybridization, Anal. Quant. Cytol. Histol. 18(5) (1996), 405-409.

[4] T. Irinopoulou, J. Vassy, M. Beil, P. Nicolopoulou, D. Encaoua and J.P. Rigaut, Three-dimensional DNA image cytometry by confocal scanning laser microscopy in thick tissue blocks of prostatic lesions, Cytometry 27 (1997), 99-105.

[5] G.D. Johnson and G.M. de C Nogueira Araujo, A simple method of reducing the fading of immunofluorescence during microscopy, Immunol. Methods 43 (1981), 349-350.

[6] A. Kriete, ed., Visualization in Biomedical Microscopies - 3-D Imaging and Computer Applications, VCH, Weinheim, 1992.

[7] J.E. McNeal, Cancer volume and site of origin of adenocarcinoma of the prostate: relationship to local and distant spread, Hum. Pathol. 23 (1992), 258-266.

[8] M.A. Micale, A. Mohamed, W. Sakr, I.J. Powell and S.R. Wolman, Cytogenetics of primary adenocarcinoma, Cancer Genet. Cytogenet. 61 (1992), 165-173.

[9] D. Pinkel, T. Straume and J.W. Gray, Cytogenetic analysis using quantitative high-sensitivity fluorescence hybridization, Proc. Natl. Acad. Sci. USA 83 (1986), 2934-2938.

[10] K. Preston, Jr., and R. Siderits, New technologies for 3-D data analysis in histopathology, Anal. Quant. Cytol. Histol. 14(5) (1992), 398-406.

[11] P.T. Scardino, R. Weaver and M.A. Hudson, Early detection of prostate cancer, Hum. Pathol. 23 (1992), $211-222$.

[12] J. Serra, Image Analysis and Mathematical Morphology, Academic Press, London, 1982.

[13] C.J.R Sheppard and M. Gu, 3-D transfer functions in confocal scanning microscopy, in: [6], pp. 251-281.

[14] P. Tekola, J.P.A. Baak, J.A.M. Beliën and J. Brugghe, Highly sensitive, specific, and stable new fluorescent DNA stains for confocal laser microscopy and image processing of normal paraffin sections, Cytometry 17 (1994), 191-195.

[15] P. Tekola, J.P.A. Baak, H.H.A.M. van Ginkel, J.A.M. Beliën, P.J. van Diest, M.A.M. Broeckert and L.T. Schuurmans, Three-dimensional confocal laser scanning DNA ploidy cytometry in thick histological sections, J. Pathol. 180 (1996), 214-222. 
[16] H. Vrolijk, W.C.R. Sloos, F.M. van de Rijke, W.E. Mesker, H. Netten, I.T. Young, A.K. Raap and H.J. Tanke, Automation of spot counting in interphase cytogenetics using brightfield microscopy, Cytometry 24 (1996), 158-166.

[17] Q. Zhu, P. Tekola, J.P.A. Baak and J.A.M. Beliën, Measurement by confocal laser scanning microscopy of the volume of epidermal nuclei in thick skin sections, Anal. Quant. Cytol. Histol. 16(2) (1994), 145-152.

[18] H. Zitzelsberger, S. Szücs, H.-U. Weier, L. Lehmann, H. Braselmann, S. Enders, A. Schilling, J. Breul, H. Höfler and M. Bauchinger, Numerical abnormalities of chromosome 7 in human prostate cancer detected by fluorescence in situ hybridization (FISH) on paraffin-embedded tissue sections with centromere-specific DNA probes, J. Pathol. 172 (1994), 325-335. 


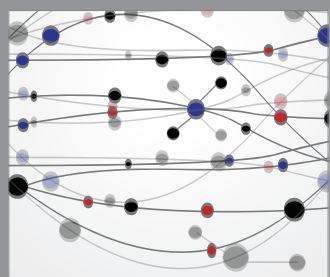

The Scientific World Journal
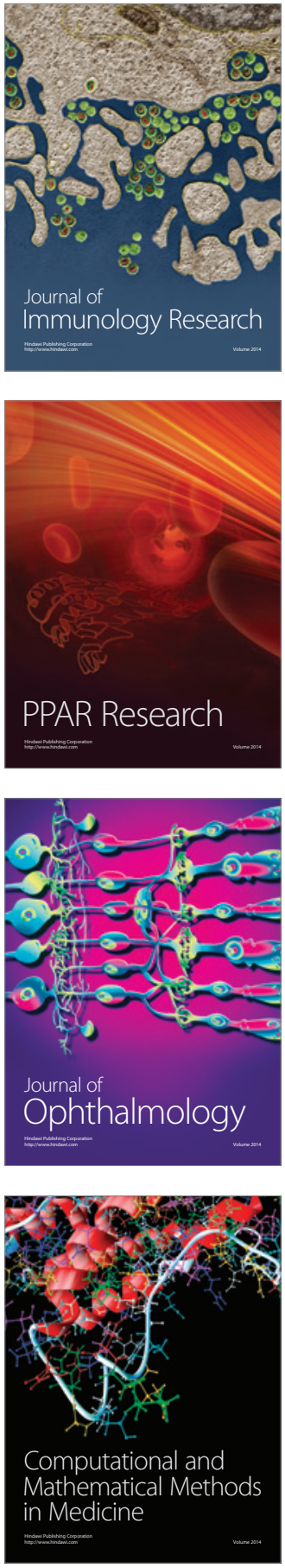

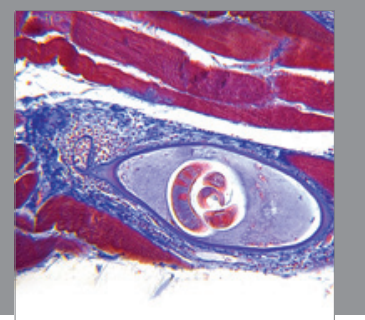

Gastroenterology

Research and Practice
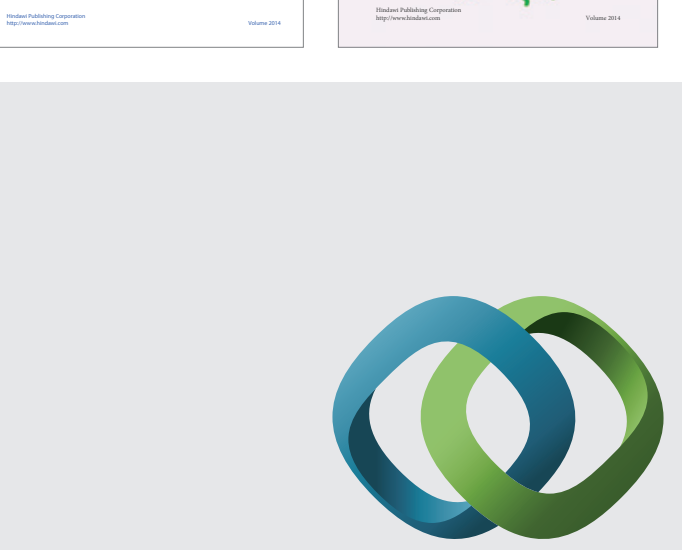

\section{Hindawi}

Submit your manuscripts at

http://www.hindawi.com
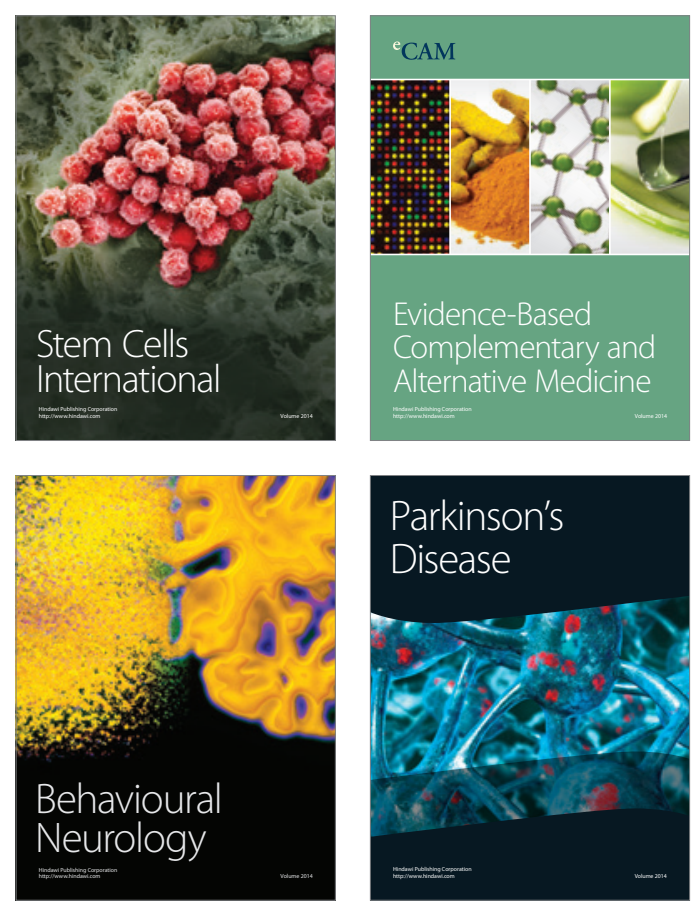

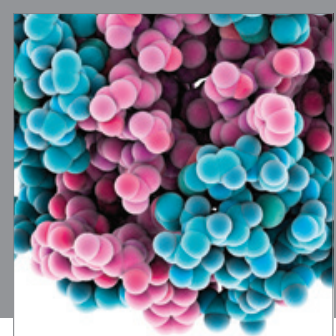

Journal of
Diabetes Research

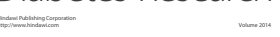

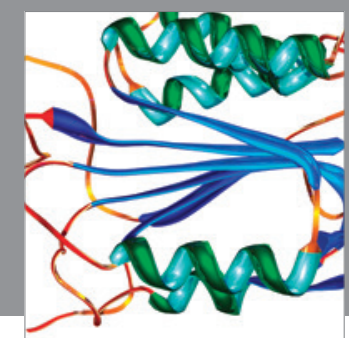

Disease Markers
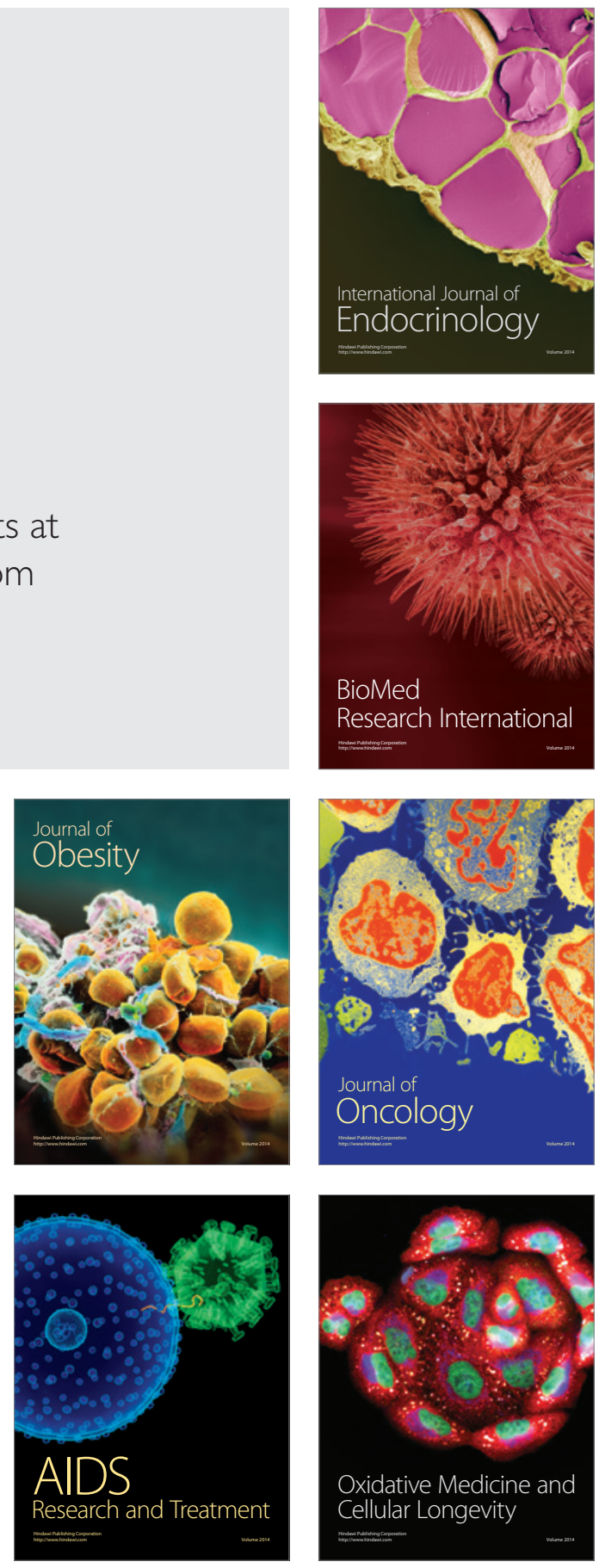